Authors (in alphabetical order by surname)

Loretta Kim (corresponding author)

School of Modern Languages and Cultures

University of Hong Kong

Pokfulam, Hong Kong SAR

Direct line: +852-3917-4251

lekim@hku.hk

* The research was conducted when L. Kim was affiliated with the History department of Hong Kong Baptist University

Rebekah Wong

University Library

Hong Kong Baptist University

Kowloon Tong, Kowloon, Hong Kong SAR

Direct line: $+852-3411-5395$

rebekahw@hkbu.edu.hk

This work was supported by the Hong Kong Baptist University Teaching and Learning Development Grant TDG/1314/06.

\begin{abstract}
This article discusses the objectives and outcomes of a project to enhance digital humanities training at the undergraduate level in a Hong Kong university. The co-investigators re-designed a multi-source dataset as an example and then taught a multi-step curriculum about gathering, organizing, and presenting original data to an introductory history course for undergraduates in a broad-based admissions social science programme. Undergraduates learned how to develop feasible topics, to search for data in different types of sources, to compile the data in spreadsheet format, and to describe the significance of the data for further research. This pilot curriculum enhanced the customary training for history students in the host institution which focuses primarily on qualitative analysis.
\end{abstract}

\title{
Keywords
}

data curation, digital humanities, history education, original data 


\section{Discovering the past through data: promoting the design and analysis of original datasets in history undergraduate courses in Hong Kong}

\section{Introduction}

Historians, like other social scientists, must be equipped to generate, manage, and interpret original data. ${ }^{1}$ Performing quantitative and qualitative analysis of datasets that are designed to be fit for purpose is all the more important for expanding historical knowledge and developing richer interpretations. Manipulating original data has become more feasible for historians in the present day because of two contextual factors. The first factor is the emerging field of digital humanities that fosters the digitalization of archival materials, advances research datasets, and promotes open access of these materials. The second is that the profession no longer solely values the achievements of individual scholars but encourages collaborative enterprises that can draw upon the strengths of several historians, or historians and specialists in other disciplines. A research group can harvest and process more data than an individual researcher, and creating datasets is a methodology that can be justifiably funded by grants.

However, a pervasive shortcoming in history education is the lack of sufficient training in data literacy and management. Without such instruction, students of history who often pursue careers in fields other than academia are ill-prepared to maximize the critical thinking, writing, and logic skills that they have cultivated through other aspects of the history disciplinary curriculum.

This paper describes and evaluates a case study carried out by the Department of History and the University Library of Hong Kong Baptist University (HKBU), with the aim of teaching first and second-year students in the Faculty of Social Sciences how to design and execute a project consisting of data collection and organization. To ensure the success of this teaching activity, the authors created a sample dataset that is now part of the Library's digital resource collection and developed a curriculum that provided step-by-step guidance to students. This project is groundbreaking because the authors know of no other undergraduate history course in Hong Kong that has approached data collection and management in this manner.

\footnotetext{
1 Original data is a set of concrete values of qualitative or quantitative variables that are created from research or generated from research sources. Original data is collected and analyzed to create or reinforce understanding or knowledge.
} 
The article will be organized in four sections. The literature review will discuss how although quantitative analysis is an important method of historical research, dataset design and management have not been common components of pedagogy in history. The project background section will introduce the research site and objectives for conducting the research. The project design part will explain how the research was carried out, and the fourth section on discussion will reflect on the benefits and areas of improvement of this pedagogical experiment.

\section{Literature review}

Performing quantitative and qualitative analysis of original data as a research method has been common throughout the historical profession, but training students, particularly undergraduates, to do so has not been a core component of history subject curricula. Students generally learn how to select primary and secondary sources of evidence, to read texts closely, and to extract details that are pertinent to their research questions. However there are few opportunities for students to learn in discipline-specific courses how to design, populate, and analyze datasets. Some may choose to develop such skills by completing courses in statistics and quantitative research in other fields such as political science, sociology, and economics.

The present condition of data analysis as an optional aspect of history training stems from the trends in attitudes towards quantitative methods. In the 1970s and 1980s, the approach of quantitative methods gained lots of attention in historical scholarship. Quantitative research extended into all branches of history. Beginning in the 1990s, this trend levelled off for a growing preference for descriptive, narrative, and prose-based approaches. Quantitative methods became dispensable in history curriculums. To restate its importance, Hudson (2000) argued that quantification is likely

to be associated with more representative and more accurate analysis than studies which are not willing to examine the characteristics of large numbers of cases.... [Quantification is also] designed to convey information or results in a standardized form which can be understood across distances of culture and class. (p. 7, 12)

Quantitative methods have the potential to connect different branches of history, create meaningful dialogues with other disciplines, and facilitate the globalization of research and academic scholarship.

Carpentier (2008) echoed the importance of quantitative methods as they could 
reinforce or sometimes challenge traditional approaches to historical research. For example, quantitative sources provided "an important response to overcome criticisms of the traditional history of education's heavy focus on the elite rather than on the masses" (p. 708). He also pointed out that a single set of quantitative sources could facilitate different uses by different disciplines in different environments, including the contemporary use of policy-making (p. 715). Nevertheless, Carpentier (2008) emphasized that quantitative and qualitative approaches should be used together, for a better and healthier development of historical scholarship (pp. 719-720).

In a more recent publication, King (2011) not only acknowledged the considerable value of data in social sciences research but, more importantly, proposed ways to ensure the availability and sustainability of data for social scientists. After discussing the possibility of setting a rule to enforce free access of data, changing scholars' mindset, and involving publishers or granting agencies, King (2011) stated "more importantly, when we teach we should explain that data sharing and replication is an integral part of the scientific process. Students need to understand that one of the biggest contributions they or anyone is likely to be able to make is through data sharing" (p. 720).

King's argument has not been realized in actual pedagogical priorities and practices. Searching through library catalogues and the internet, the authors found nine student's guides published in the recent eight years, although some of them were a revised version of an earlier edition. These nine books were Abbott (2009), Barber and Peniston-Bird (2009), Benjamin (2013), Bombaro (2012), Elder, Gorzycki, and Paul (2011), Lukacs (2014), Mabbett (2007), Storey (2013), and Williams (2012). They discussed examination preparation, writing process and skills, research skills, libraries and historical sources, information searching and evaluation, citation styles, critical thinking in history, narrative techniques, employability, among other topics.

Content about data sources, data analysis, data creation, data preservation, and data sharing was much less than for these topics. Four out of these nine books did not discuss data at all (Barber \& Peniston-Bird, 2009; Lukacs, 2014; Mabbett, 2007; Storey, 2013). Another four touched on statistical information. Abbott (2009) and Williams (2012) briefly mentioned a need for statistics and population trends in the research process (p. 42; pp. 70-72). Benjamin (2013) cited multiple dataset resources in the Appendix, including a dedicated section on "Historical Statistics" (pp. 221-224). Bombaro (2012) highlighted the importance of statistical information and provided a detailed list of books, subscription-based databases, and free websites with historical 
statistics (pp. 50-56). Only one book addressed data as one of its major topics. Elder, Gorzycki, and Paul (2011) asserted that "we use concepts, ideas and theories to interpret data, facts, and experiences in order to answer questions, solve problems, and resolve issues.... All historical reasoning is based on DATA, INFORMATION, and EVIDENCE" (pp. 33, 42). However, data resources provided by these authors were highly limited; most, if not all, data were related to the contemporary history of political, economic, and social areas. Moreover, none of these student's guides taught undergraduate readers how to create, use, preserve, and share data.

Turning the focus on classroom teaching, the authors attempted to find case studies that described how individual history professors integrated knowledge of data use and data management into their courses. The results of this inquiry were similarly limited as with the history curricular guides. Only a few examples of applying data were found. One is in 1992, Miller and Lekander reported success in developing the Great American History Machine, a system that enabled history undergraduates to explore a huge amount of census and election data of the United States for the nineteenth and twentieth centuries (p.4). Miller and his team also designed a teaching activity, facilitating students to explore six classic problems in American history using the data provided by the system. The purpose of this project was to empower undergraduates to "think like historians", being able to "search for patterns in huge bodies of information" (Miller \& Lekander, 1992, p.5).

A second example is Davies, Conneely, Davies, and Lynch (2000), who identified "numerical data" as one of the four particular areas of interest and importance in their new curriculums when their history courses were re-designed (p. 113). The first data exercise was delivered in a seminar in which small groups of students were asked to identify and discuss gaps in a dataset relating to the British General Election in 1992. It was hoped that the new course structure could help students enhance their confidence in dealing with data.

The authors could not find any relevant case studies that were written in the latest decade. The overview of existing literature showed that data use and data management were rarely taught in history courses, especially in recent years. The current article will fill some of the gaps by providing a recent example of how data use and data management can be integrated into a history curriculum.

\section{Background of the project}


As a medium-sized public higher institution, HKBU positions itself as a broad-based education provider in Hong Kong through offering programs in a variety of areas including arts, business, Chinese medicine, communication, social sciences, science, and visual arts. The course in which the data management was taught was an introductory course given by the History department for first and second-year undergraduates in the Faculty of Social Sciences. The primary objective of the course was to teach students joining the Faculty about what history is as a subject of advanced study and academic research, and how it differs from history as taught in primary and secondary schools. For the first two years in which the course was given, the curriculum focused on introducing theories of historical analysis, types of history such as public history and family history, and famous historians and their achievements.

A comprehensive assessment of the curriculum revealed that most students were not interested in learning about such specific aspects, especially since most of them would major in other disciplines such as Government and International Studies, Sociology, and Geography. Therefore, one of the co-authors, who led the first version and became the course head for the third year's version, decided to teach students how historians conduct their research. This immersion method, which would empower them to be "practicing historians," was considered to be more meaningful for both students, who only took this one course in history, and for those who would choose history as their major. The former would gain skills that would be applicable to other majors, and the latter would develop a solid foundation for taking intermediate and advanced history courses.

This course was important as a part of a major development in the Hong Kong educational system and one at HKBU. The Hong Kong-wide change was from most undergraduate degree programs, excluding professional training in fields such as medicine, being structured for completion in three years to four years. The "3-3-4 scheme," meaning that students would be in lower secondary school (Forms 1-3) for three years, upper secondary school (Forms 4-6) for three years, and undergraduate degree programs for four years, was implemented starting in 2009. The principal impact on universities was that students would be entering with one less year of secondary education and be younger than students who had finished the previous "3-4-3" course. Adjustments were made to all curricula for first-year students to accommodate their relative disadvantage in knowledge and maturity. HKBU concurrently changed the admissions system in which students started their majors in the Faculty of Social Sciences from year one to having a first year in which students 
would take General Education courses and then apply for admission to majors during the summer between their first and second years. The rationale for this development was that students could explore all the majors by taking introductory courses and electives offered by all the Social Sciences departments.

Almost simultaneously, the University Library went through a major organizational reform, with the aim of providing necessary campus support for data management and digital humanities as a new strategic direction for the library. A new Digital and Multimedia Services Section in the Library was formed to help faculty members enhance their research data collection methodologies and engage in digital data publishing. The Library welcomed any opportunities to collaborate with faculty to develop this direction. When these two seemingly unrelated activities - the curriculum change of a history course and the new library direction-were linked together, they provided an essential structure for the development of this project.

The two co-authors (and project co-investigators) are a historian of late imperial and modern China and a librarian serving at HKBU. The historian was a member of the history department at HKBU from 2010 to 2014. During that period, she taught undergraduate courses on the history of modern Asia, ethnic studies in post-1949 China, Sino-Russian relations from early modern to contemporary times, and gender issues in China's imperial era. Her major research interests were and continue to be the regional history of Northeast China, China's borderland administration system during the Qing dynasty (1644-1911 CE), and ethnic minority languages and cultures in the Amur River region. As of this article's publication, she is a member of the School of Modern Languages and Cultures faculty at the University of Hong Kong. The Digital and Multimedia Services Librarian at HKBU, carrying the title of senior assistant librarian, leads the library's initiatives on digital humanities, data management, and scholarly communication. The historian co-author (hereby known as "the historian") worked with a "history team" consisting of one research assistant to help with the first step of the project, which was data collection, and the second step, which was teaching a course, with another teaching assistant. The librarian co-author (hereby known as "the librarian") supervised a "library team" to help standardize the dataset and transform it into a web-based database.

\section{Project design}

The project consisted of two parts. The first step of the process was the re-organization and enrichment of the historian's dataset so that it could serve as an 
example for the students (see Figure 1). This step also fell into the library's broader effort to provide digital humanities support to faculty. The library team collaborated with the history team to fill incomplete entries, check and remove duplicated records, separate single records as necessary and provide linkage among relevant data, design and use controlled vocabularies, among other tasks. The library team contributed their experience of dataset organization and the history team contributed their subject knowledge to this collaboration. The library team then developed a digital humanities website "History in Data: Tribute Data Curation" (http://digital.lib.hkbu.edu.hk/history/tribute.php?lang=en), transforming the dataset into five interactive charts and tables that can be further researched in many different ways (see Figure 2). The full dataset is also available on a publicly accessible website. The first step took both teams nine months to complete.

Figure 1 here

Figure 2a here

Figure $2 b$ here

Figure $2 \mathrm{c}$ here

The second phase of the project was using the dataset and the website as a model for teaching first-year students how to create datasets that can be interpreted by quantitative and qualitative analysis. The authors created a curriculum that not only taught practical information about how to organize a dataset, but also the significance of using quantifiable data in historical research systematically and comprehensively. The curriculum was based on the assumption that students had no prior relevant experience. It consisted of ten modules, each teaching a different skill including how to designate a topic for the dataset, to identify sources of raw data, to standardize the presentation of elements such as numbers and proper nouns, and to analyze the content of the dataset once completed.

Each module was taught in 50 minutes, which was half the time of each class meeting, and divided into multiple segments The specific allocation of time for each part of an individual module, which ranged from 5 to 15 minutes in duration, depended on the skill being taught. The general principle for designing each lesson plan was that students would learn by applying the featured skill to their own data rather than just by listening to a lengthy explanation about it by the instructor. For example, for the module on converting spreadsheet data into graphs, students worked with their group members to prepare samples of their datasets before the lesson and then after hearing the instructor and teaching assistant introduce the technical skills needed to create 
graphs and compare examples of useful and problematic graphs, they worked on their own computers to produce graphs. The instructor and teaching assistant then selected two groups to present their graphs to the rest of the class and explain which variables determined their data sub-sets and which kind of graph they chose to represent that data.

The two authors of this article also co-taught some of the modules. For one class meeting, the librarian gave a guest lecture, explaining the differences between qualitative and quantitative research, the importance of quantitative research, ways to collect data, criteria and benefits of data sharing, as well as standards and tips of data organization. This lecture made use of real examples taken from the first phase of the project. A data exercise was also conducted right after the lecture, asking students to work in groups and identify mistakes in another dataset relating to film history. The instructor and teaching assistant contributed feedback on the guest lecture PowerPoint content and the data exercise before the guest lecture to make sure the material was suitable for these students. Correspondingly, the librarian advised the history team about good practices in dataset development, so that the history team could manage the daily operations of the course and give problem-specific support to the students.

Students completed datasets as written assignments at the end of the semester. Working in groups of ten to twelve, they divided up the work of finding relevant sources, compiling and organizing data, and writing project reports. Each project report included the dataset in spreadsheet form, descriptions of the dataset parameters and methodology for identifying sources, analysis of the dataset content, and significance of the dataset for research and practical applications. The dataset topics were: the influences of the Silk Road on Chinese economy and society during the Ming and Qing dynasties, the Beiyang Navy in the late nineteenth century, composition of the HK Legislative Council (1980-97), Red Guards in the Chinese Cultural Revolution (1966-76), films in 1990s Hong Kong, public housing in HK (1960s to present), Japanese industrial textile development, Jesuit missionaries in China, the impact of Mikhail Gorbachev's policies on the Soviet Union's economy and society, and effect of the Industrial Revolution on women's economic status in Great Britain (1760-1840).

The projects were evaluated based on three criteria: fulfillment of basic requirements, such as including all the aforementioned sections and formatting the project according to the mandatory style standards, accuracy and precision of the data, and consistency of content presentation in the dataset, and finally creativity and originality applied to 
topic selection, data analysis, and interpretation of the dataset's significance.

The quantity and quality of data in the projects varied significantly. Some groups compiled substantial amounts of data, 200 to 500 rows in a spreadsheet, because they identified and extracted content from many sources. Others created datasets with less evidence, about 50 to 100 rows in a spreadsheet, primarily because there were fewer sources available for their topics.. Differences in quality were also apparent in the datasets as presented in spreadsheets and other forms such as tables and graphs. Some groups collected and organized their data based on multiple variables. Others focused solely on one or two variables, such as time and geographical location.

Manipulating data was meaningful to students encountering history as it is taught at the university level because they could link together diverse factors influencing a given historical phenomenon. Before taking the course, most of the students only considered history as a subject requiring the memorization of facts. They realized by seeing how elements such as territorial demarcation, financial resources, and population size change that history is not about past occurrences that are inevitable, but that are produced by ongoing and mutable processes. Students saw "history in motion" by organizing and discerning patterns in their datasets.

\section{Discussion}

This case study yielded three types of results. The first was how teaching dataset management can enrich the learning experience for an introductory history course. The second was how students benefit from developing basic competency in dataset creation and analysis. The third was the synergy between research and teaching that was achieved through the overhaul of the sample dataset during the project's first phase. Finally, the co-authors identified areas of improvement for students who will be learning dataset management in future versions of the course.

\section{Enhancement of learning}

The new curriculum diverged from the conventional mode of learning and teaching history at HKBU. Students worked differently from the usual practice, in which they pursue assigned topics, find relevant evidence from secondary sources, and then describe the results of analyzing the evidence utilizing prescribed methods. Alternatively, students had to think of innovative topics based on their own interests, to find sources independently, to generate datasets, and then to analyze not only the 
evidence but also to argue why their datasets are significant resources for exploring original research topics.

Working in groups for one semester, students mastered the basic skills of these tasks. All of the ten groups produced datasets that reflected their particular academic interests and demonstrated their ability to standardize information from multiple sources into custom-designed frameworks. As this mode of learning and teaching history was new to students, the authors found that it was particularly useful to start this kind of training at the beginning of their undergraduate study. The students had more time and more opportunities to become competent in data management and independent in research.

\section{Lasting benefits to students}

This project aligns with the HKBU History department's general objective to equip students with a wide variety of tools and skills so that they may conduct research using appropriate methodologies. It also fulfills the Social Sciences faculty's aim of providing a common foundation for junior (first and second-year) students that will help them succeed in their third and fourth-year studies, irrespective of major.

Many undergraduates majoring in, or just taking courses in, history do not learn how to utilize datasets, much less formulate ones that they can use for original research. Students enrolled in the first-year course learned from the hands-on curriculum that they do not have to rely on existing datasets, but can also develop fit-for-purpose collections. As only one-fourth of these students will be history majors, this change of mindset and the data skills that they learned will eventually be reflected and applied in other Social Sciences courses as well as courses that they take to complete General Education requirements and electives in other faculties.

The greater implications of this learning experience will most likely manifest itself in two or three years when they will be writing final-year projects, postgraduate theses, or even the work they will do in a variety of non-academic and professional settings, such as government service or business analysis. They will then have the option to create datasets individually, rather than as groups, that will aggregate evidence about diverse topics.

\section{Reinventing a research dataset}


This project revamped a dataset that was previously only usable by the creator, the historian, into a version that can be used, expanded, and modified by a variety of users. The website that publishes and visualizes this dataset can be accessed through the library's homepage as one of the library's digital humanities projects. As mentioned earlier, the website displays five interactive charts and tables that are generated from the dataset. Users can set and modify chart parameters to generate new charts. Website users can also retrieve the full dataset or part of the dataset based on the parameters they set. The whole website and the dataset are available in English and traditional Chinese, supplemented with a Chinese-English-Manchu glossary.

The website had 14,775 views between its launch in September 2014 and May 2015. Although it is impossible to identify the background of each individual user as the website is publicly accessible, statistics showed a wide diversity of user demographics. Website users came from Hong Kong, China, Taiwan, Russia, United States, South Korea, Macau, Germany, United Kingdom, and Japan. 40 percent of users were new visitors; 60 percent were returning visitors. The use statistics indicated that the project fulfilled the goal of making the dataset content accessible to the scholarly community at large.

\section{Reflection and resolutions for future improvement}

Students had several opportunities to provide feedback about their learning experiences. The history team spoke with students before and after each class meeting, and answered questions about the dataset design curriculum by email and other indirect means throughout the semester. Students were also invited to reflect upon their own performance as part of the reports accompanying the final datasets. These sessions were not evaluated but were reviewed carefully by the history team during the assessment process.

Many of the student comments were about the difficulty of adopting a new, unfamiliar mode of learning. Almost all students started the course without any knowledge of how datasets are developed and did not understand how or why datasets can be used as tools and resources for research. They acknowledged that the greatest challenge in studying the curriculum was to change their own thinking about what is a research source and why data must be organized systematically and thoroughly (rather than "cherry-picking" select facts) in order to be useful for objective analysis. Some students expressed preferences for what they considered to be "traditional research" (reading texts, identifying and reiterating evidence relevant to given topics), while 
others enjoyed the opportunity to select their own subjects (rather than instructors' assigning them) and to present their data in creative ways.

The co-authors believe that the greatest challenge in this project, maximizing student interest in dataset management, is also the key to further development of the curriculum and teaching-learning methods. Student feedback about individual modules will be considered if and when the curriculum content is edited. Some modules may require more supporting materials such as visual step-by-step guides, rubrics, and samples that students can manipulate while learning a skill. Other modules may be divided into discrete parts that will mean that students must successfully complete each one in order.

Another major form of innovation will be to create elements of dataset management learning that can be incorporated into other history courses, particularly those that teach advanced research skills. This change will reduce the perception that the original course is simply exceptional and that students will have no further use for what they have learned about datasets. Having dataset management be taught incrementally and throughout the undergraduate years will also help students discover how they can use the skills not only for conducting research but also for future study and employment that are not related to history.

\section{Conclusion}

The project succeeded in accomplishing its main goal that was to teach students the significance of quantifiable data in historical research and basic data management skills, through a sample dataset, a re-developed curriculum, lectures, and a course assignment. There were challenges but also clear opportunities. It was particularly encouraging that some students conquered "data phobia" and became more confident in their ability to work with data. These students learned that they can do basic quantitative analysis without having advanced ability in numerical sciences. They also discovered that they can go beyond the usual comfort zone of culling and repeating evidence from other scholars, and generate their own evidence through quantitative methods.

Another important outcome of the project is a rewarding relationship between the academics and librarians. Through tackling a teaching-learning challenge, a strengthened partnership between the two departments developed. This project also generated a good model of symbiotic partnership — the academics provide subject 
expertise, research data, and co-teaching opportunities, and librarians provide support for data management and digital humanities for both faculty and students - for other faculty members to follow. The authors hope that this kind of faculty-library collaboration will continue, so that students of various disciplines as future researchers can be trained to be competent and independent in harvesting, organizing, analyzing, and sharing data.

\section{Acknowledgements}

The authors would like to thank the University Library and the History Department of Hong Kong Baptist University, Mr. Haipeng Li, Dr. Eva Wong of the Centre of Holistic Teaching and Learning at Hong Kong Baptist University, Mr. Patrick Ho-tung Ho, and Mr. Login Lok-yin Law. 


\section{References}

Abbott, M. (Ed.). (2009). History skills: A student's handbook. New York: Routledge.

Barber, S., \& Peniston-Bird, C. M. (Eds.). (2009). History beyond the text: A student's guide to approaching alternative sources. New York, NY: Routledge.

Benjamin, J. R. (2013). A student's guide to history. Boston, MA: Bedford/St. Martin.

Bombaro, C. (2012). Finding history: Research methods and resources for students and scholars. Lanham, MD: Scarecrow Press.

Carpentier, V. (2008). Sources and interpretations: Quantitative sources for the history of education. History of Education, 37(5), 701-720.

Davies, P., Conneely, J., Davies, R., \& Lynch, D. (2000). Imaginative ideas for teaching and learning. In A. Booth \& P. Hyland (Eds.), The practice of university history teaching (pp. 112-24). Manchester: Manchester University Press.

Elder, L., Gorzycki, M., \& Paul, R. (2011). Student guide to historical thinking. Tomales, CA: Foundation for Critical Thinking.

Hudson, P. (2000). History by numbers: An introduction to quantitative approaches. London, England: Arnold.

King, G. (2011, February 11). Ensuring the data-rich future of the social sciences. Science, 331, 719-721.

Lukacs, J. (2014). A student's guide to the study of history. Wilmington, DE: ISI Books.

Mabbett, I. W. (2007). Writing history essays: A student's guide. New York, NY: Palgrave Macmillan.

Miller, D., \& Lekander, B. (1992). Extending and disseminating a new method of teaching U.S. history. Retrieved from ERIC database. (ED 414884)

Storey, W. K. (2013). Writing history: A guide for students. Oxford, England: Oxford 
University Press.

Williams, R. C. (2012). The historian's toolbox: A student's guide to the theory and craft of history. Armonk, NY: M.E. Sharpe.

\section{Figure captions}

Figure 1: A Snapshot of the Original Dataset as Presented in a Spreadsheet Figure 2a: Home Page of "History in Data: Tribute Data Curation" Website Figure 2b: Sample Line Graph of "History in Data: Tribute Data Curation" Website Figure 2c: Sample Bar Graph of "History in Data: Tribute Data Curation" Website 
Figure 1a

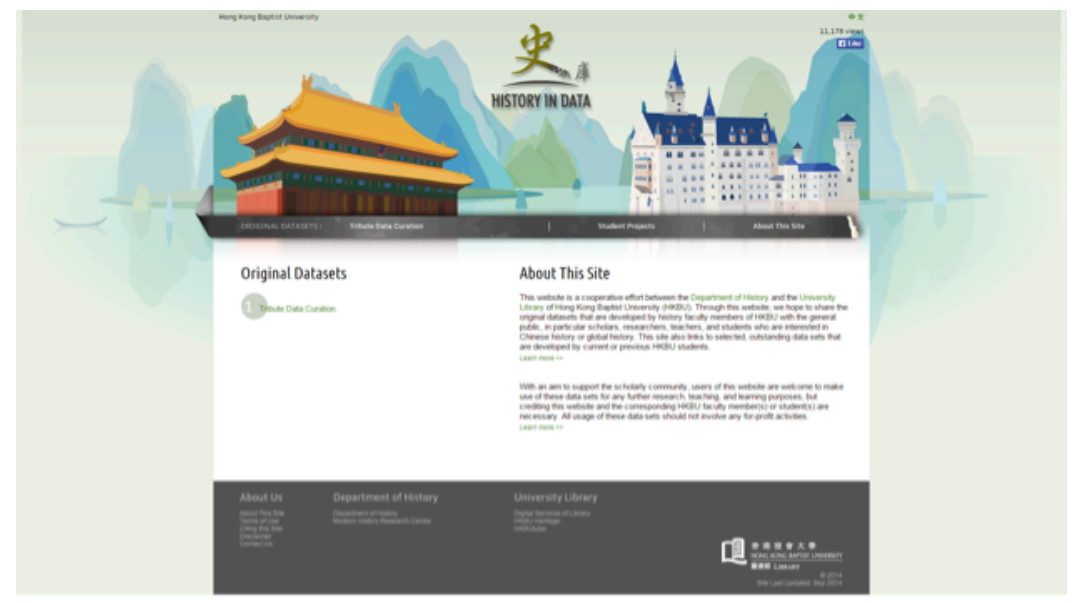

Figure $1 b$

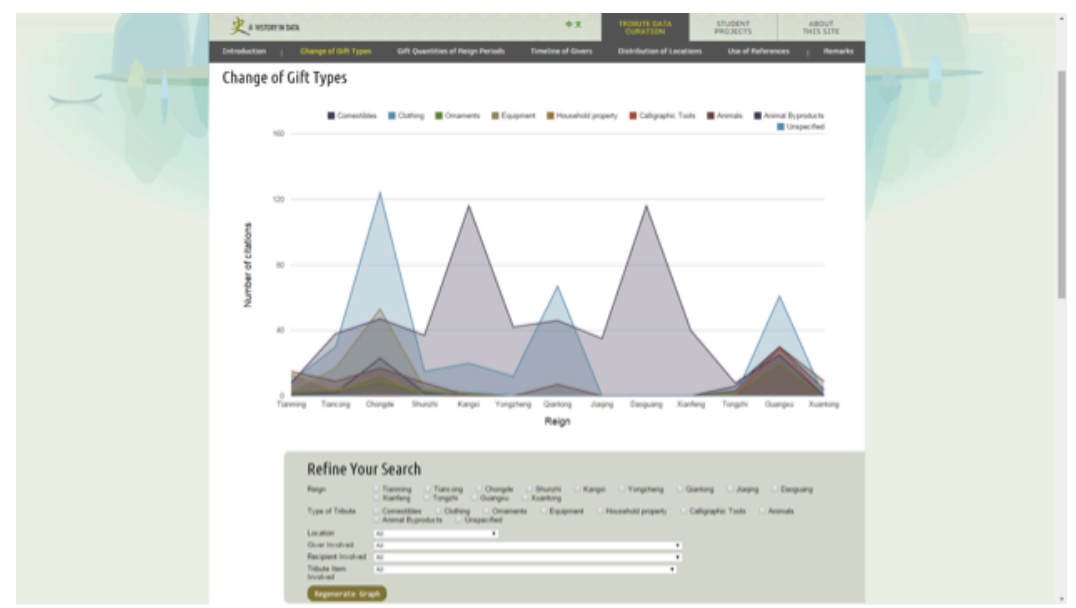

Figure 1c

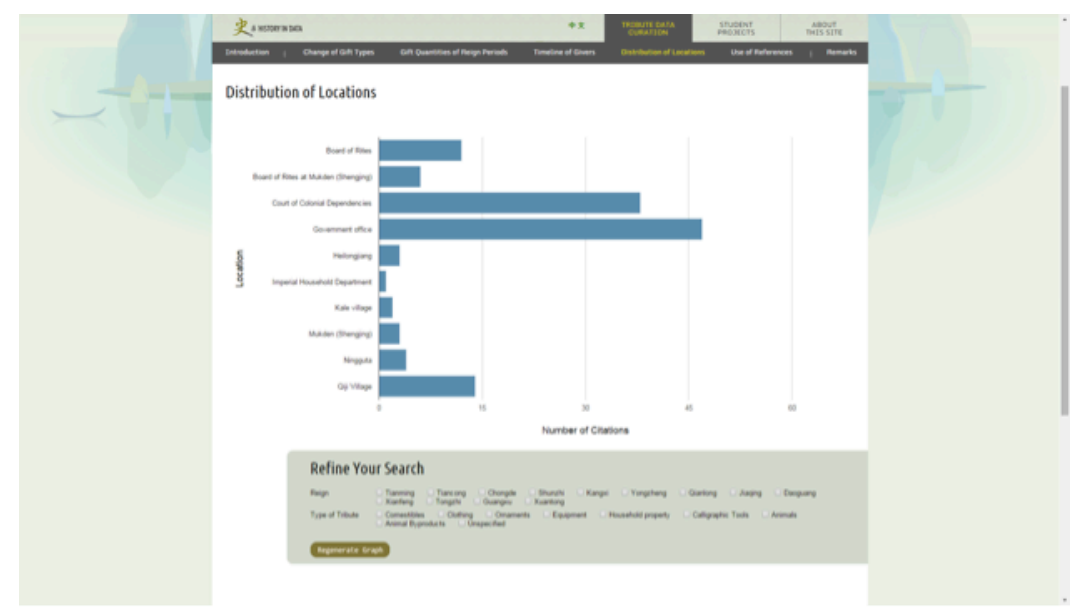

